\title{
Treatment of Established Peritoneal Fibrosis by Gene Transfer of Smad7 in a Rat Model of Peritoneal Dialysis
}

\author{
Yanyan Sun $^{\mathrm{a}}{\text { Fengxin } \mathrm{Zhu}^{\mathrm{a}} \text { Xueqing } \mathrm{Yu}^{\mathrm{a}} \text { Jing Nie }{ }^{\mathrm{a}} \text { Fengxian Huang }}^{\mathrm{a}}$ \\ Xiaoyan $\mathrm{Li}^{\mathrm{a}}$ Ning Luo $^{\mathrm{a}}$ Hui Yao Lan ${ }^{\mathrm{b}}$ Yongtao Wang ${ }^{\mathrm{a}}$ \\ a Department of Nephrology, The First Affiliated Hospital, Sun Yat-Sen University, Guangzhou, Guangdong, and \\ ${ }^{b}$ Department of Medicine, University of Hong Kong, Hong Kong, SAR, PR China
}

\section{Key Words}

Gene therapy $\cdot$ Smad7 $\cdot$ Peritoneal fibrosis $\cdot$ Peritoneal

dialysis $\cdot$ TGF- $\beta 1 \cdot$ Ultrasound

\begin{abstract}
Background/Aims: It has been shown that blockade of TGF$\beta 1$ signaling with Smad7 prevents experimental peritoneal fibrosis. The present study investigated whether Smad7 has a therapeutic effect on established peritoneal fibrosis associated with peritoneal dialysis (PD). Methods: A rat model of peritoneal fibrosis was induced by a daily intraperitoneal infusion of $4.25 \%$ Dianeal. After peritoneal fibrosis had been established on day 14, groups of 6 rats were treated intraperitoneally with gene transfer of Smad7 or control plasmids using an ultrasound-microbubble-mediated system for 2 weeks until day 28. In addition, a group of 6 diseased rats was euthanized on day 14 before treatment as the basal disease control. Results: Compared to the control-treatment animals on day 28, real-time PCR, Western blot, and confocal microscopy revealed that Smad7 gene transfer significantly attenuated the increased peritoneal fibrosis including the thickening of fibrotic peritoneum, accumulation of $\alpha$-SMA and collagen $\mathrm{I}$, and an improvement in peritoneal dysfunc-
\end{abstract}

\section{KARGER}

Fax +41613061234 E-Mail karger@karger.ch www.karger.com

\section{(c) 2009 S. Karger AG, Basel}

0250-8095/09/0301-0084\$26.00/0

Accessible online at:

www.karger.com/ajn tion (all p < 0.05). Importantly, Smad7 treatment also improved the severity of peritoneal fibrosis and functional impairment when compared to those on day 14 before treatment (all $p<0.05$ ). Inhibition of the established peritoneal fibrosis by Smad7 was associated with an abrogation of TGF$\beta$ signaling and upregulation of TGF- $\beta 1$ and PAI-1. Conclusions: Smad7 gene therapy is able to inhibit established peritoneal fibrosis in a rat model of PD. Results from this study suggest that Smad7 may be a therapeutic agent for the treatment of peritoneal fibrosis associated with PD.

Copyright $\odot 2009$ S. Karger AG, Basel

\section{Introduction}

Continuous ambulatory peritoneal dialysis (CAPD) is an attractive option for the treatment of patients with end-stage renal disease. However, peritoneal fibrosis induced by the recurrent peritonitis and hyperglycemic dialysis solutions ultimately results in ultrafiltration failure and therefore limits the use of CAPD as renal replace-

Y.S. and F.Z. contributed equally to this work.
Prof. Xueqing Yu

Department of Nephrology, The 1st Affiliated Hospital, Sun Yat-Sen University 74 Zhongshan Road II

Guangzhou, Guangdong 510089 (PR China)

Tel. +86 208733 5811, Fax +862087776 9673, E-Mail yuxq@mail.sysu.edu.cn 
ment therapy [1-4]. Thus, it is important to develop new therapeutic strategies by specifically targeting mediators or pathways responsible for peritoneal fibrosis.

Increasing evidence has shown that transforming growth factor (TGF)- $\beta$ is a key mediator of experimental and human peritoneal fibrosis associated with peritoneal dialysis (PD) [5]. In vitro, peritoneal mesothelial cells are the major source of TGF- $\beta 1$ production in response to high glucose, which leads to extracellular matrix (ECM) accumulation by increasing the synthesis of ECM proteins while reducing their degradation $[6,7]$. In long-term CAPD patients, the concentrations of TGF- $\beta 1$ in PD effluents are significantly elevated, particularly in those with peritonitis $[8,9]$. Direct evidence for the pathogenic role of TGF- $\beta$ comes from the finding that peritoneal fibrosis can be induced by the deliberate overexpression of TGF- $\beta 1$ after adenovirus-mediated gene transfer in rats $[10,11]$.

Recent studies have suggested a pivotal role for Smads as intracellular effector molecules of the TGF- $\beta$ family members. TGF- $\beta$ induces fibrosis through activation of the downstream signaling pathway such as $\operatorname{Smad} 2 / 3$ [12]. In addition, activation of TGF- $\beta$ signaling can also result in the expression of inhibitory Smads including Smad7, which inhibits $S \operatorname{mad} 2 / 3$ phosphorylation by blocking their access to TGF- $\beta$ receptors or causing degradation of TGF- $\beta$ receptors via a negative feedback mechanism [13]. Thus, overexpression of Smad7 can inhibit TGF- $\beta$ induced $S m a d 2 / 3$ activation and fibrosis in vitro in renal tubular epithelial cells and mesangial cells, and in vivo in a number of kidney disease models including obstructive and remnant kidney disease [14-18] and autoimmune nephritis [19]. Furthermore, we and other investigators have also shown that overexpression of peritoneal Smad7 is capable of inhibiting peritoneal fibrosis and inflammation induced by chronic PD in both uremic and non-uremic rats [20-22]. However, it remains unknown whether blockade of TGF- $\beta /$ Smad signaling with Smad7 has a therapeutic effect on the established peritoneal fibrosis, which was investigated in the present study in an established rat model of PD.

\section{Materials and Methods}

Ultrasound-Mediated Gene Transfer of Inducible Smad7 Gene into the Peritoneum

An endotoxin-free, flag-tagged pTRE-m2Smad7 plasmid and an improved pEFpurop-Tet-on plasmid were prepared using the Qiagen plasmid mega kit (Qiagen Inc., Valencia, Calif., USA) and were mixed with Sonovue (echocardiographic contrast microbubble; Bracco s.p.a., Milan, Italy) at a ratio of 1:1 (v/v) as described previously $[21,22]$. Following the established protocol of gene transfer of inducible Smad7 into the peritoneal cavity [21, 22], the mixed solution containing $900 \mu \mathrm{g}$ of designated plasmids in a total volume of $6 \mathrm{ml}$ was injected into the abdominal cavity. Immediately after injection, the ultrasound transducer (Ultax, Fysiomed Sonic15, Belgium) was directly applied to the abdominal skin surface coated with the ultrasound media with a continuous wave output of $1 \mathrm{MHz}$ at $10 \%$ power output for a total of $10 \mathrm{~min}$. Then the animal was turned over and treated with the ultrasound again for another $10 \mathrm{~min}$. The same procedure was also applied to the control animals that received the mixed solution containing the same amount of empty control plasmids (pTRE and Tet-on).

\section{Animal Model}

An animal model of PD associated with peritoneal fibrosis was induced following the established protocol as described previously $[21,22]$. Briefly, 18 male Sprague-Dawley rats, weighing $160-$ $170 \mathrm{~g}$, were treated with daily intraperitoneal injection of $4.25 \%$ Dianeal (Baxter Healthcare, Deerfield, Ill., USA) at a dose of 100 $\mathrm{ml} / \mathrm{kg}$ and lipopolysaccharide (LPS; Sigma, St. Louis, Mo., USA) at a dose of $0.6 \mathrm{mg} / \mathrm{kg}$ in a 3-day interval over a period of 14 days. All animals were randomly allocated into 3 groups ( $n=6$ rats in each group) on day 14 as: (1) a disease model group on day 14 (PD14); (2) an empty vector-control group on day 28 (VC), and (3) a Smad7-treated group on day 28 (Smad7). Rats in the disease model group (PD14) were euthanized on day 14 as the basal disease control before treatment. In the Smad7-treated group, rats were treated with a mixture of pTRE-m2Smad7/pEFpurop-Tet-on plasmids plus Sonovue followed immediately by ultrasound irradiation as described above. The same procedure was applied to the vector control-treated animals, except for substitution of pTRE-m2Smad7 with empty vectors. To induce Smad7 transgene expression, $1 \mathrm{ml}$ of doxycycline (Sigma) at a concentration of 200 $\mu \mathrm{g} / \mathrm{ml}$ was administered into the abdominal cavity in both control and Smad7-treated animals immediately after ultrasoundmicrobubble gene transfer, followed by the addition of doxycycline in the daily drinking water $(200 \mu \mathrm{g} / \mathrm{ml})$ for 14 days until being euthanized on day 28. In addition, a group of 6 age-matched normal rats was used as a normal control.

Assessment of the Smad7 Gene Transfection Rate

To determine the Smad7 gene transfection rate in the fibrotic peritoneum after PD, 30 additional rats treated with $4.25 \%$ Dianeal and LPS for 14 days were transfected with the mixture solution of the Flag-tagged-pTRE-m2Smad7 and Tet-on plasmids using an ultrasound-mediated technique as described previously $[21,22]$. Groups of 6 rats were sacrificed on day 14 before Smad7 gene transfer and on days 17,21,24, and 28 after gene transfection. The Smad7 transfection rate and transgene expression within the fibrotic peritoneum were examined by both immunofluorescence and Western blot with the anti-Flag-m2 antibody (Sigma) as described previously [21, 22].

Peritoneal Function Test

Peritoneal functional tests were performed as previously described $[21,22]$. Briefly, for the peritoneal ultrafiltration rate, $4.25 \%$ Dianeal at $0.09 \mathrm{ml} / \mathrm{g}$ body weight was administered intra- 
peritoneally to the rats before being euthanized. Four hours later, the peritoneal fluid was removed for ultrafiltration measurement. Net ultrafiltration was the volume of fluid removed after $4 \mathrm{~h} \mathrm{mi-}$ nus the volume of fluid administered. For glucose transportation assay, glucose was measured by a standard enzymatic test on a Hitachi automated chemistry analyzer (Hitachi 7170, Japan). Mass transfer of glucose from the peritoneum was calculated using the formula: (initial dialysate glucose initial volume) - (final dialysate glucose final volume). These values were corrected for animal weights at the time of euthanasia.

\section{Histology and Confocal Immunofluorescence}

Four-micrometer paraffin sections from the anterior abdominal wall were stained with Masson's trichrome. The thickness of the peritoneal membrane was measured in sections as previously described [22]. Each section was measured at 10 random sites. The thickness $(\mu \mathrm{m})$ of the peritoneum was measured in each animal using a micrometer fitted into the eyepiece of the microscope and expressed as the means $\pm \mathrm{SD}$.

For confocal imaging analysis, $6-\mu \mathrm{m}$ paraffin sections were pretreated with citrate buffer $(10 \mathrm{mM}, \mathrm{pH} 6.0)$ for $13 \mathrm{~min}$ at $37^{\circ} \mathrm{C}$ and then incubated with the indicated antibodies at $4^{\circ} \mathrm{C}$ overnight. Sections were then stained with FITC-labeled goat antimouse or goat anti-rabbit antibody, mounted with a DAPI (Sigma) nuclear stain and examined under confocal microscopy (Zeiss LSM-510, Oberkochen, Germany). Images were taken using Zeiss LSM-510 Confocal Imaging System.

\section{Western Blot Analysis}

Protein was extracted from frozen visceral peritoneal tissues by homogenization in a standard lysis buffer with addition of proteinase inhibitors and then transferred to a nitrocellulose membrane for Western blot analysis as previously described [21, 22]. Briefly, after blocking with 5\% skimmed milk, the membranes were incubated for $1 \mathrm{~h}$ with the antibody to TGF- $\beta 1$ (Chemicon, Temecula, Calif., USA), Smad7 (Santa Cruz Biotechnology, Santa Cruz, Calif., USA), flag-m2 (Sigma), Smad2/3, phospho-Smad2/3 (Santa Cruz Biotechnology), $\alpha$-SMA (Sigma), PAI-1 (Santa Cruz Biotechnology), type I collagen (Santa Cruz Biotechnology), and glyceraldehyde-3-phosphate dehydrogenase (GAPDH; Santa Cruz Biotechnology). After washing, the membranes were incubated with the secondary antibody such as peroxidase-conjugated anti-mouse IgG or anti-rabbit IgG. The signals obtained were visualized by an enhanced chemiluminescence system (GE, Piscataway, N.J., USA) and the ratio was calculated by subjecting to GAPDH.

\section{RT-Polymerase Chain Reaction}

Total peritoneal RNA was isolated from visceral peritoneum using Trizol Reagent (Invitrogen Life Technologies, Carlsbad, Calif., USA) and was reverse-transcribed using RevertAid ${ }^{\mathrm{TM}}$ First Strand cDNA Synthesis Kit (MBI Fermentas Inc., Glen Burnie, Md., USA) as previously described [21, 22]. Amplified cDNA was used as the template DNA and PCR was performed with Taq DNA polymerase (Promega, Madison, Wisc., USA) and specific primers. The primers used in this study for TGF- $\beta 1, \alpha$-SMA, collagen I, and PAI-1 were as described previously [21]. Samples were analyzed on a $1.2 \%(\mathrm{w} / \mathrm{v})$ agarose gel, visualized by ethidium bromide, and photographed using a gel 1000 ultraviolet documentation system (Bio-Rad, Hercules, Calif., USA). All samples were sub- jected to RT-PCR for housekeeping gene GAPDH for densitometry.

\section{Statistical Analyses}

Data obtained from this study are expressed as means \pm SD. Differences in ultrafiltration volume, mass transfer of glucose, and peritoneal thickness, and differences in Smad7, Flag-m2, $\alpha-$ SMA, TGF- $\beta 1$, p-Smad2/3, type I collagen, and PAI-1 expression within and between the groups were assessed by one-way analysis of variance followed by a Tukey's comparison program (a t test between groups) from GraphPad Prism 3.0 (GraphPad Software, San Diego, Calif., USA). A p value of $<0.05$ denoted the presence of statistically significant difference.

\section{Results}

\section{Expression of Exogenous Smad7 Transgene within the} Fibrotic Peritoneum Tissue

We have previously shown that the ultrasound-microbubble mediates a high rate of gene transfection in normal peritoneal tissues $[21,22]$. We now examined the efficacy of $\mathrm{m} 2 \mathrm{Smad} 7$ transgene expression in fibrotic peritoneum tissues over the period of 14-28 days after PD. As shown in figure 1 , following gene transfer on day 14 , both confocal and Western blot analyses showed that the ultrasound-microbubble mediated high levels of Smad7 gene transfection rate and transgene expression, resulting in more than $80 \%$ of peritoneal cells being Flag-m2positive on day 17 (fig. 1A). Similar results were also found by Western blot with high levels of Flag-m2Smad7 protein expression (fig. 1B). This was associated with a marked increase in total peritoneal Smad7 expression throughout the entire disease course (fig. 1C). There was no detectable $\mathrm{m} 2 \mathrm{Smad} 7$ in normal peritoneal tissues and in parenchyma tissues of liver, heart, lung, spleen, kidney, and gastrointestinal tissues after ultrasound-microbubble-mediated gene transfer (data not shown).

\section{Effect of Overexpression of Smad7 on Peritoneal \\ Histology}

Morphologic changes were assessed in tissue sections stained with Masson's trichrome. In normal animals, the peritoneal tissue consisted of a monolayer of mesothelial cells and exiguity of connection tissues under the mesothelial layer (fig. 2A). A marked thickening of the submesothelial zone with the presence of fibrosis was found on day 14 after peritoneal infusion (fig. 2B). Animals treated with control plasmids showed progressive thickening of peritoneal tissues on day 28 (fig. 2C). In contrast, animals treated with gene transfer of Smad7 exhibited a significant reduction in peritoneal thickening when compared 
A Flag-m2Smad7 immunofluorescence (confocal)
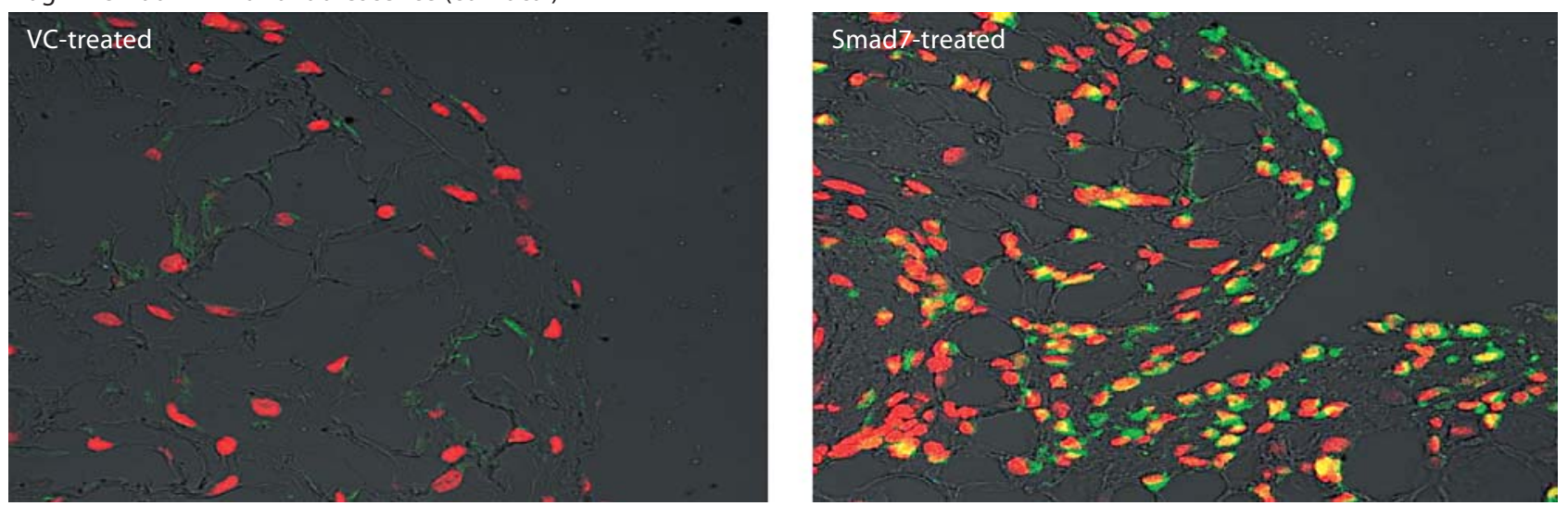

B Flag-m2Smad7 transgene expression

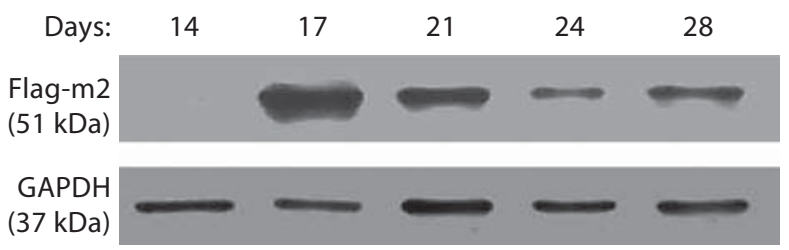

C Total Smad7 expression
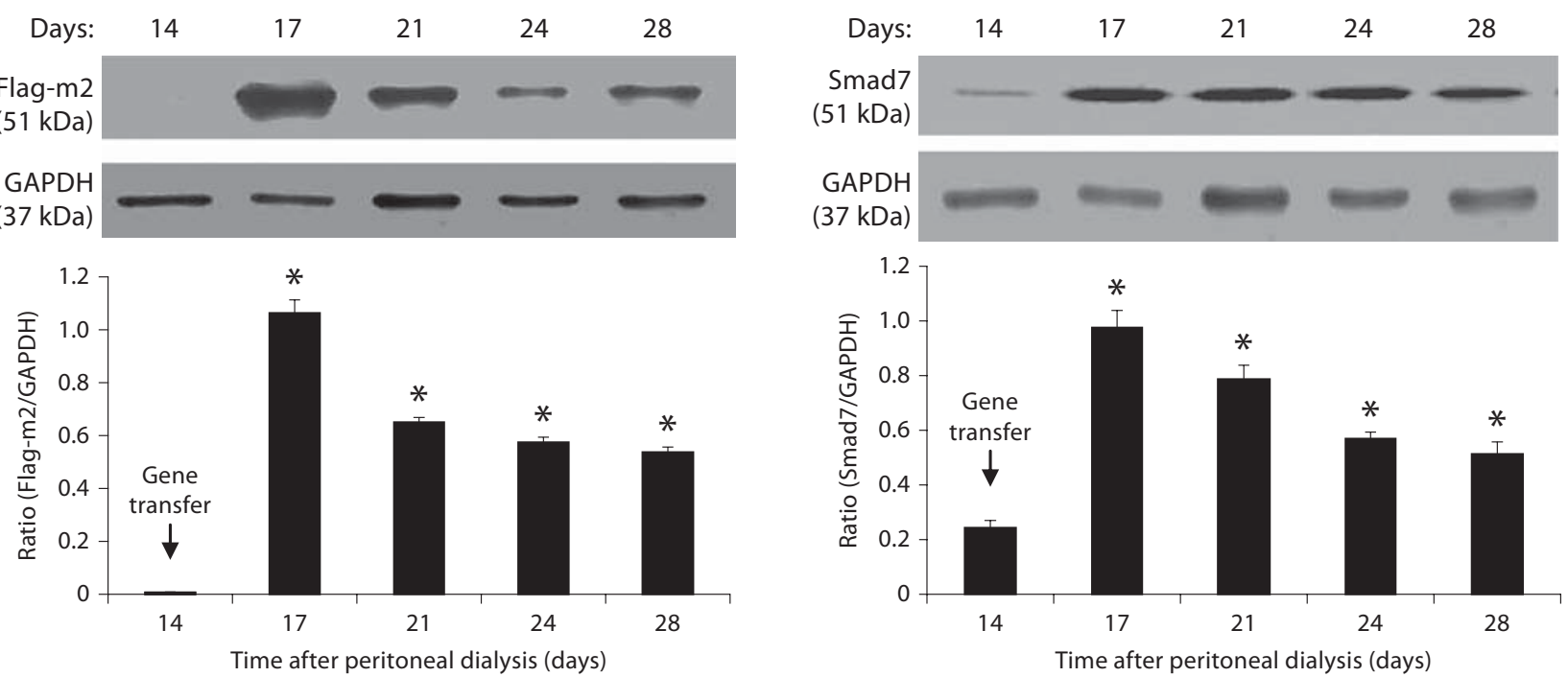

Fig. 1. Ultrasound-microbubble-mediated Smad7 transfection rate and transgene expression in fibrotic peritoneal tissues. A Dox-regulated flag-m2-Smad7 pTRE plasmid and a Tet-on plasmid (1:1) were mixed with Sonovue at a ratio of 1:1 (vol:vol) and transfected into the diseased peritoneum on day 14 after PD by the ultrasound-microbubble-mediated system. Smad7 transgene expression was detected by confocal microscope and Western blot with the anti-Flag-M2 antibody as described in the Methods. A Confocal microscopy shows a strong Flag-m2Smad7 expression (green) in mesothelial cells on the peritoneal surface(s) and also in cells in submesothelial areas on day 3 after gene transfer (day
17 of the PD model), accounting for up to $80 \%$ of peritoneal cells, but this is negative in the control vector treatment. $\times 400$. B Western blot analysis demonstrates Flag-m2Smad7 transgene expression over the 2-week period after gene transfer (days 14-28 of the PD model). C Western blot analysis of total peritoneal Smad7 expression over days 14-28 after gene transfer on day 14 of the PD model. Note that there is a minor Smad7 expression in the diseased peritoneal tissues on day 14 (C), which is largely increased after gene transfer of Smad7. Data represent the mean \pm SD for groups of 6 rats. ${ }^{*} \mathrm{p}<0.01$ vs. day 14 group. Nuclei were counterstained with DAPI (red). to those on day 14 before treatment and those treated with control plasmids on day 28 , although peritoneal fibrosis remained apparent when compared to normal peritoneal tissue (fig. 1D). This was confirmed by quantitative analysis as shown in figure $2 \mathrm{E}$.

Smad7 Inhibits Established Peritoneal Fibrosis in Rats

\section{Overexpression of Smad7 Attenuates Progressive} Peritoneal Function Injury

As shown in figure 3, compared to normal animals, severe peritoneal dysfunction as demonstrated by a significant decrease in the peritoneal ultrafiltration rate and 

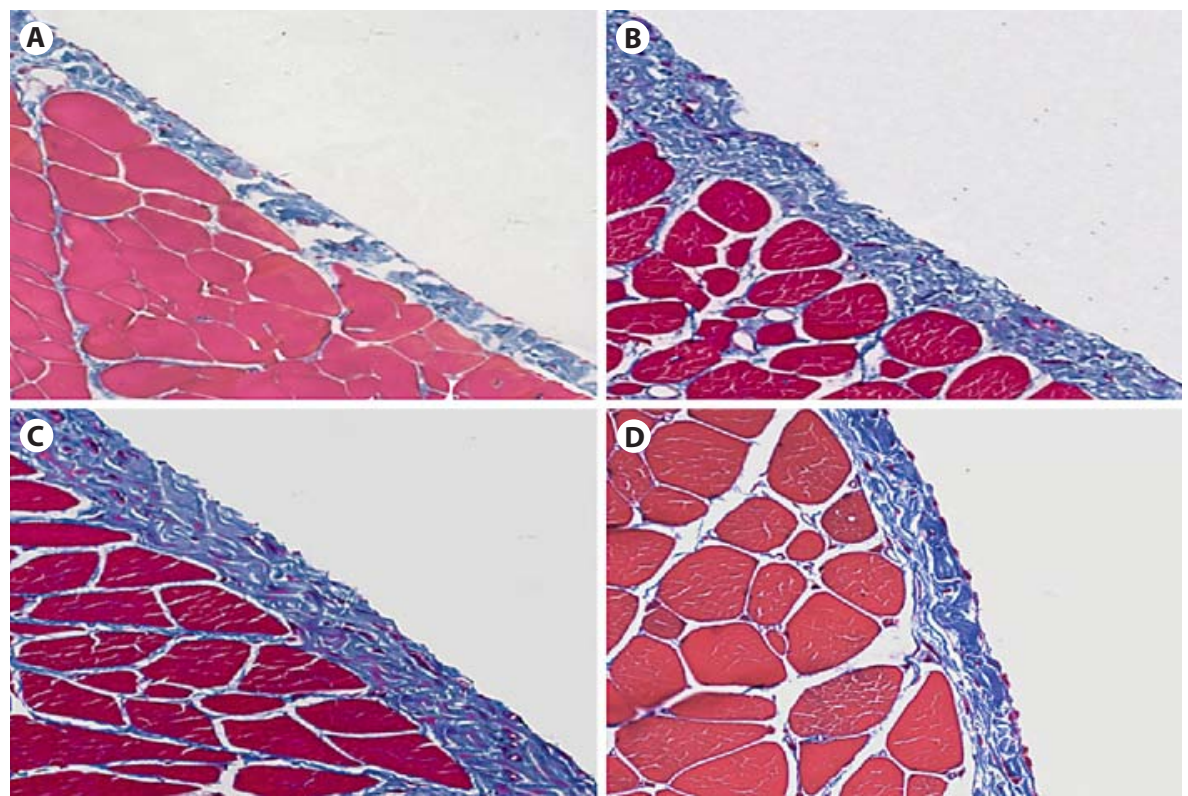

Fig. 2. Effect of Smad7 gene therapy on peritoneal histology after PD examined by Masson's trichrome staining. $\times 200$. A Normal control (N). B A PD rat on day 14 before gene transfer (PD14). C A PD rat on day 28 with control vector treatment (VC). D A PD rat on day 28 with Smad7 treatment (Smad7). E Quantitative analysis. Data represent mean $\pm \mathrm{SD}$ for groups of 6 rats. ${ }^{*} \mathrm{p}<0.01$ compared to the agematched normal control $(\mathrm{N}) ;{ }^{*} \mathrm{p}<0.05$ compared to both untreated PD on day 14 (PD14) and vector control treatment on day 28 (VC).

E Quantitative analysis

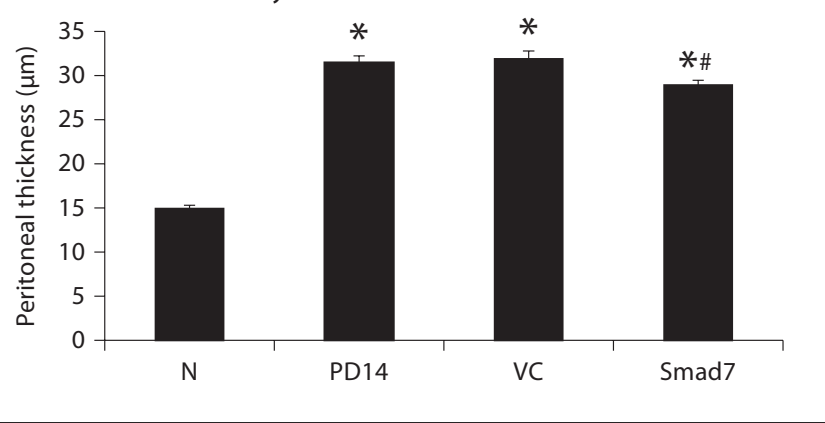

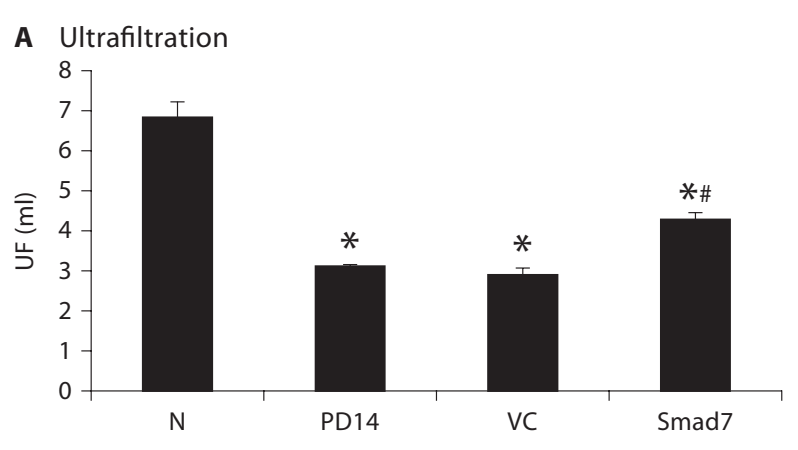

Fig. 3. Overexpression Smad7 inhibits peritoneal dysfunction. A The ultrafiltration rate (UF). B Mass transfer of glucose (MTG). Data represent mean \pm SD for groups of 6 rats. ${ }^{*} \mathrm{p}<0.05 \mathrm{com}-$
B Glucose transportation

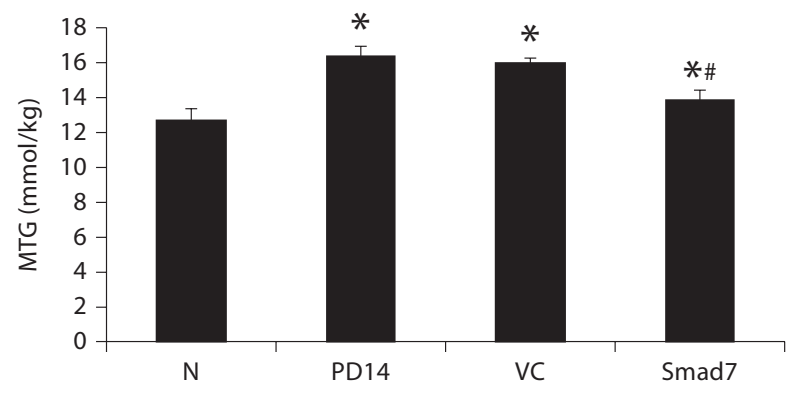

pared to the age-matched normal control $(\mathrm{N}) ;{ }^{\#} \mathrm{p}<0.05$ compared to both untreated PD on day 14 (PD14) and vector control treatment on day 28 (VC). 


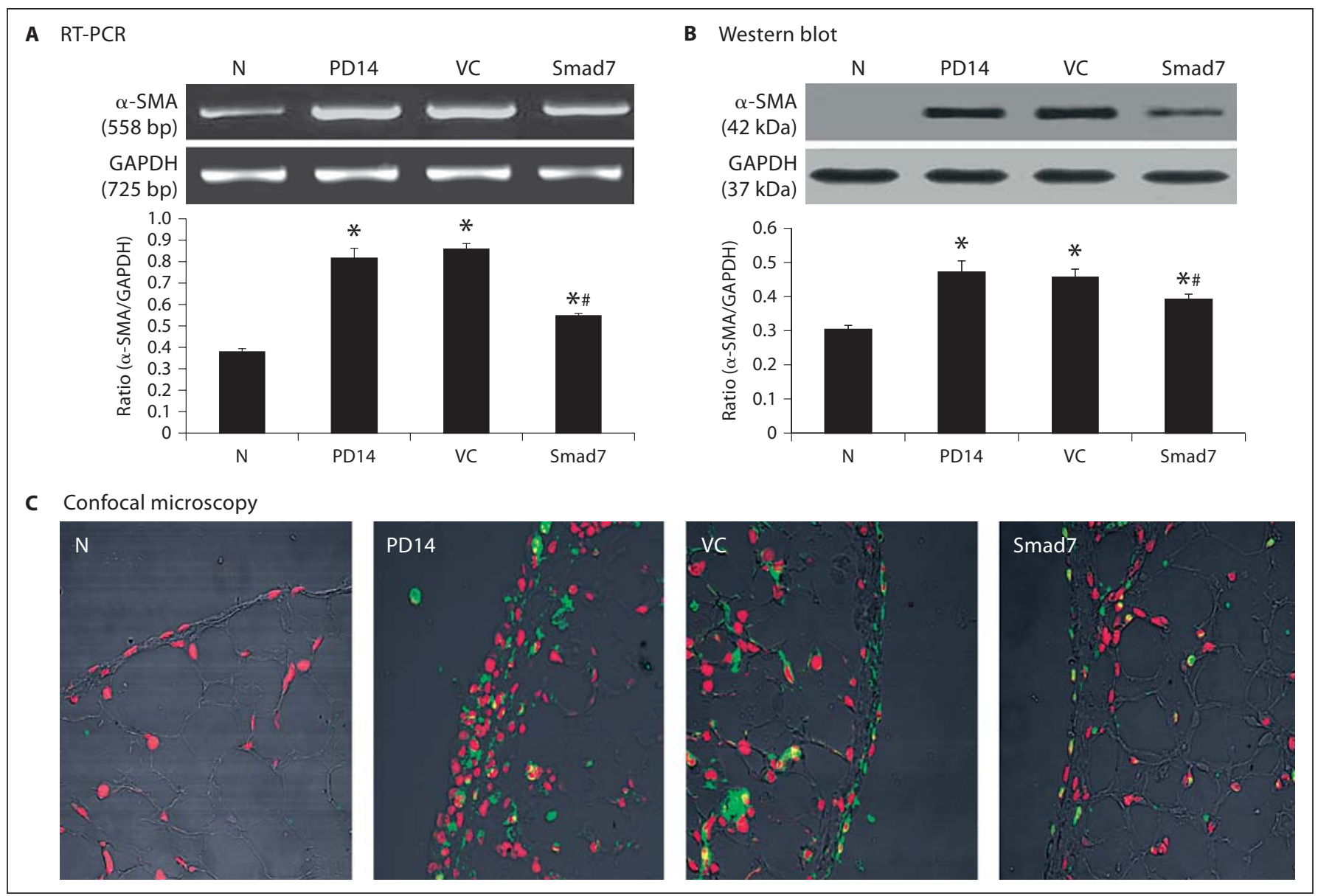

Fig. 4. Smad7 gene transfer inhibits $\alpha$-SMA mRNA and protein expression in a rat model of peritoneal fibrosis. $\mathrm{N}=$ Normal animals; PD14 $=$ PD rats on day 14 before treatment; $\mathrm{VC}=\mathrm{PD}$ rats treated with empty plasmids on day $28 ; \operatorname{Smad} 7=\mathrm{PD}$ rats treated with Smad7 gene transfer on day 28. All analyses reveal that Smad7 treatment inhibits $\alpha$-SMA mRNA and protein expression when compared to diseased animals on day 14 before treatment or on day 28 after treatment. Data represent mean \pm SD for groups of 6 rats. ${ }^{*} \mathrm{p}<0.05$ compared to the age-matched normal control; ${ }^{\#} \mathrm{p}<0.05$ compared to both PD14 and VC animals. A RTPCR. B Western blot analysis. C Confocal microscopy. Nuclei are counterstained with DAPI (red). $\times 400$. an increase in the mass transport of glucose was found in animals after a 14-day peritoneal infusion. Treatment with control vectors from day 14 to 28 did not alter the severity of peritoneal dysfunction. However, Smad7 treatment significantly improved the impaired peritoneal function when compared to those treated with control empty vectors on day 28 (fig. 3). Interestingly, Smad7 treatment also reversed the peritoneal functional injury when compared to diseased animals on day 14 before treatment, but it did not recover to the normal level (fig. 3).
Smad7 Gene Therapy Improves Established Peritoneal Fibrosis

As shown in figures 4 and 5, RT-PCR, Western blot, and confocal immunofluorescent examinations revealed that all animals developed severe peritoneal fibrosis as demonstrated by a significant increase in $\alpha$-SMA and collagen I mRNA and protein expression on day 14 after PD. Consistent with changes in histology and peritoneal function, treatment with control vectors from day 14 to 28 did not alter high levels of peritoneal $\alpha$-SMA and collagen I expression when compared to diseased animals on day 14. In contrast, overexpression of peritoneal Smad7 not only attenuated upregulation of peritoneal $\alpha$-SMA

Am J Nephrol 2009;30:84-94 


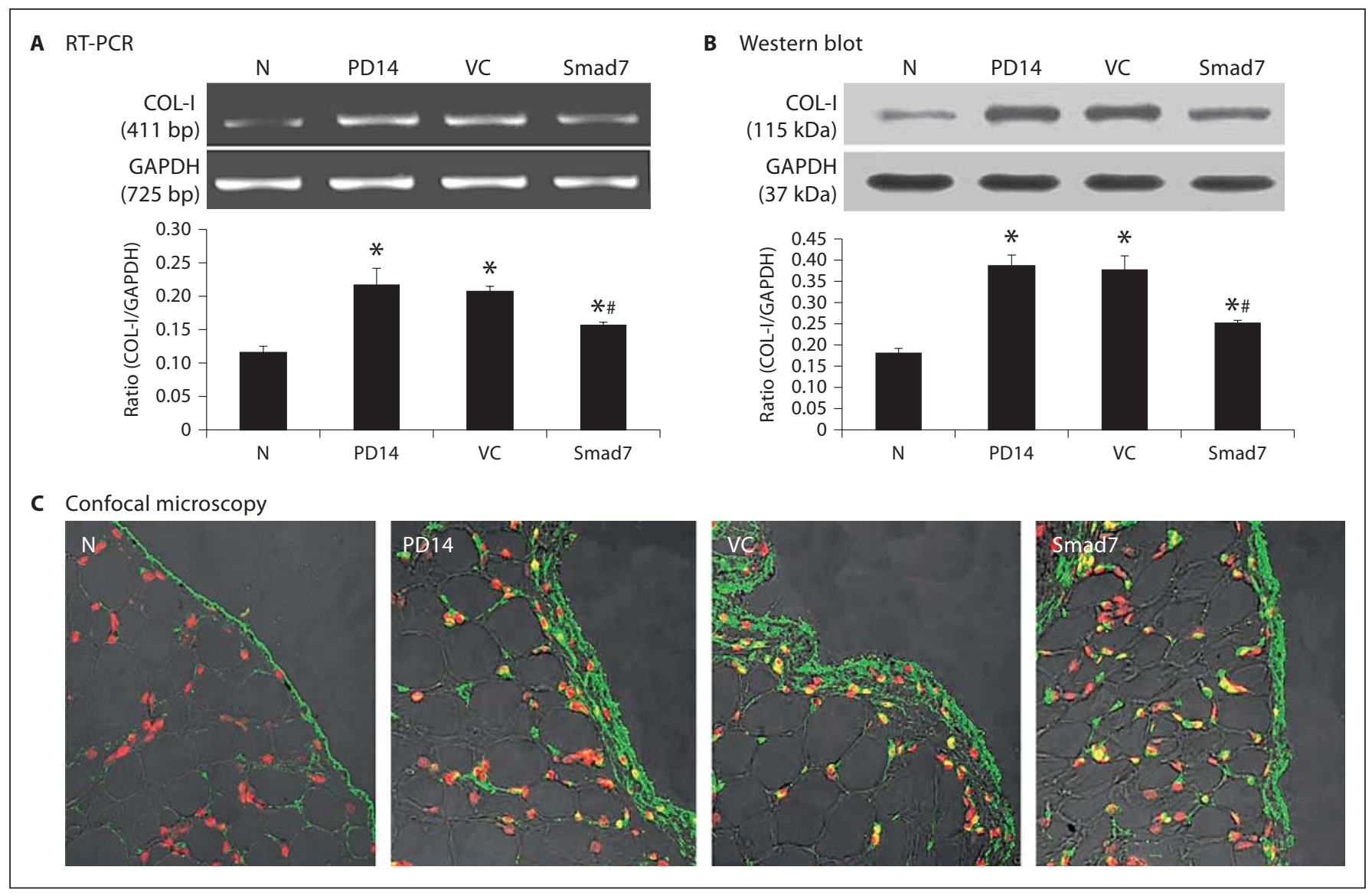

Fig. 5. Smad7 gene transfer blocks collagen I mRNA and protein expression in a rat model of peritoneal fibrosis. $\mathrm{N}=$ Normal animals; PD14 $=$ PD rats on day 14 before treatment; $\mathrm{VC}=\mathrm{PD}$ rats treated with empty plasmids on day $28 ; \mathrm{Smad} 7=\mathrm{PD}$ rats treated with Smad7 gene transfer on day 28. All analyses reveal that Smad7 treatment inhibits collagen I mRNA and protein expres- sion when compared to diseased animals on day 14 before treatment or on day 28 after treatment. Data represent mean \pm SD for groups of 6 rats. ${ }^{*} \mathrm{p}<0.05$ compared to the age-matched normal controls; ${ }^{*} \mathrm{p}<0.05$ compared to both PD14 and VC animals. A RT-PCR. B Western blot analysis. C Confocal microscopy. Nuclei are counterstained with DAPI (red). $\times 400$. and collagen I mRNA and protein expression compared to diseased animals treated with control empty vectors on day 28 , but also significantly inhibited their expressions when compared to those on day 14 before treatment (fig. 4, 5). It should be pointed out that Smad7 treatment from day 14 to 28 was unable to completely reverse upregulated $\alpha$-SMA and collagen I when compared to the age-matched animals (fig. 4,5 ).

\section{Mechanisms by Which Smad7 Gene Transfer Inhibits \\ Established Peritoneal Fibrosis}

As shown in figures 6 and 7, further studies by RTPCR, immunohistochemistry, and Western blot analyses demonstrated that inhibition of the established perito- neal fibrosis by overexpressing Smad7 on day 28 was associated with blockade of peritoneal TGF- $\beta 1$ and PAI-1 expression when compared to both control animals on days 14 and 28.

We have previously shown that activation of TGF- $\beta$ / Smad signaling is critical in peritoneal fibrosis and overexpression of peritoneal Smad7 is capable of inhibiting activation of Smad2/3 and peritoneal fibrosis [21]. Thus, we examined whether overexpression of Smad7 is capable of inhibiting the activated $\operatorname{Smad} 2 / 3$ by Western blot and immunohistochemistry using the anti-phosphorylated Smad2/3 antibody. As shown in figure 8 , there was a marked activation of $S m a d 2 / 3$ in the peritoneum on day 14 after dialysis fluid infusion, which remained high 
A $\quad$ RT-PCR
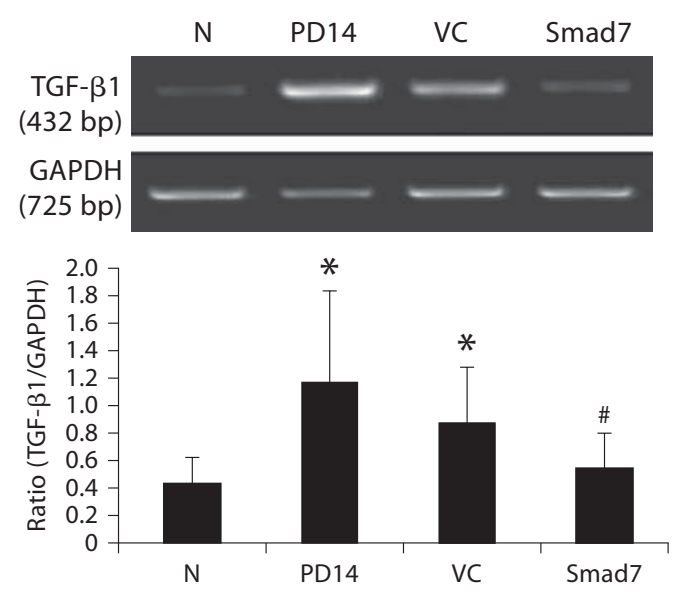

C Confocal microscopy
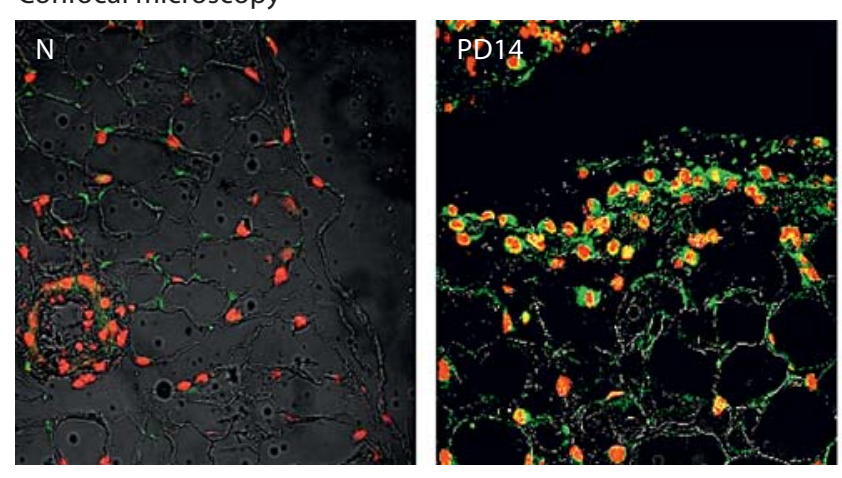

B Western blot
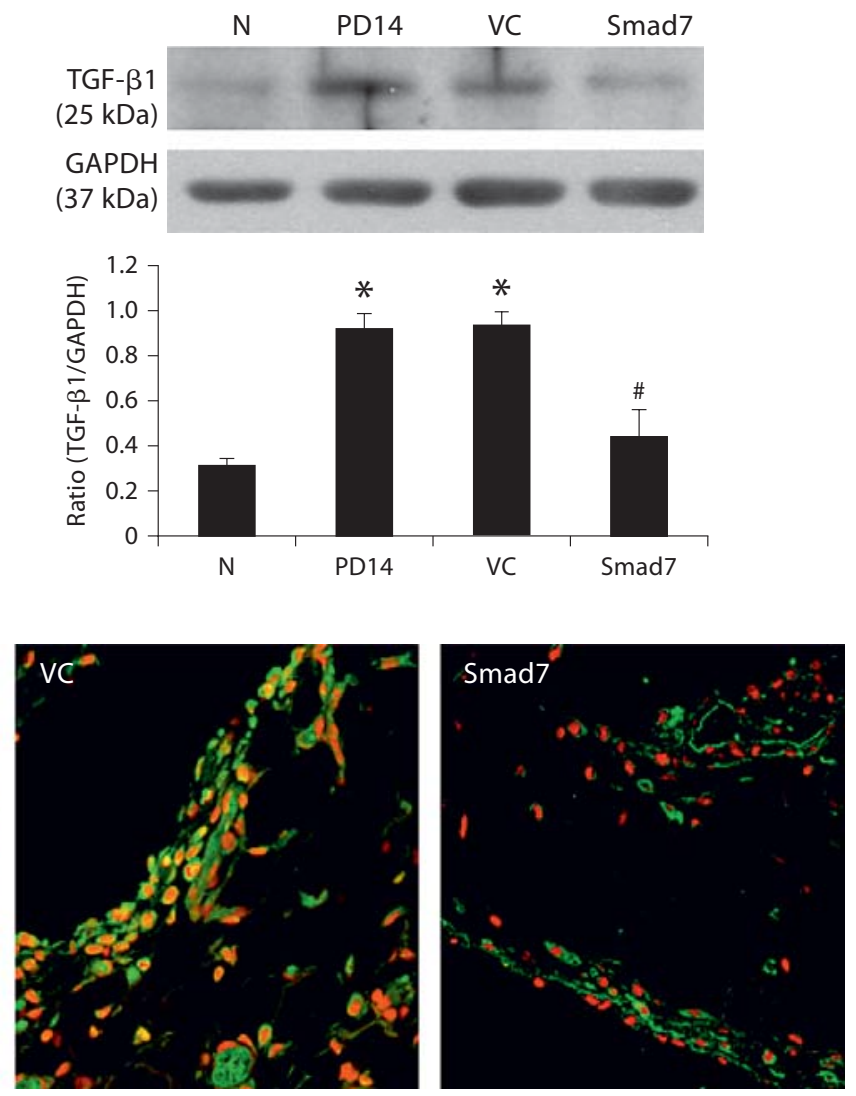

Fig. 6. Smad7 gene transfer suppresses TGF- $\beta 1$ I mRNA and protein expression in a rat model of peritoneal fibrosis. $\mathrm{N}=$ Normal animals; $\mathrm{PD} 14=\mathrm{PD}$ rats on day 14 before treatment; $\mathrm{VC}=\mathrm{PD}$ rats treated with empty plasmids on day 28; Smad7 $=$ PD rats treated with Smad7 gene transfer on day 28. Results show that Smad7 treatment blocks TGF- $\beta 1 \mathrm{mRNA}$ and protein expression when compared to diseased animals on day 14 before treatment or on day 28 after treatment. Data represent mean \pm SD for groups of 6 rats. ${ }^{*} \mathrm{p}<0.05$ compared to the age-matched normal controls; ${ }^{\#} \mathrm{p}<0.05$ compared to both PD14 and VC animals. A RT-PCR. B Western blot analysis. C Confocal microscopy. Nuclei are counterstained with DAPI (red). $\times 400$. throughout the 28-day disease course in animals that received control vector treatment. In contrast, Smad7 gene transfer was able to block the activated TGF- $\beta / S$ mad signaling when compared to diseased animals before treatment on day 14 and to those treated with control vectors on day 28 (fig. 8).

\section{Discussion}

This study demonstrates that gene transfer of Smad7 into the established peritoneal fibrosis tissues from day 14 to 28 after a chronic PD was able to inhibit the progression of peritoneal fibrosis and peritoneal functional in- jury. Interestingly, Smad7 gene transfer also improved the severity of peritoneal fibrosis and functional impairment when compared to diseased animals on day 14 before treatment. This was demonstrated by the findings that Smad7 gene transfer to rats with established peritoneal fibrosis from day 14 for 2 weeks not only reduced the thickening of the peritoneal membrane and upregulation of $\alpha$-SMA and type I collagen mRNA and protein on day 28 , but also significantly improved these fibrotic changes when compared with those before treatment on day 14 . These findings imply that gene therapy with Smad7 could potentially be a useful clinical treatment method for peritoneal fibrosis. 
A RT-PCR
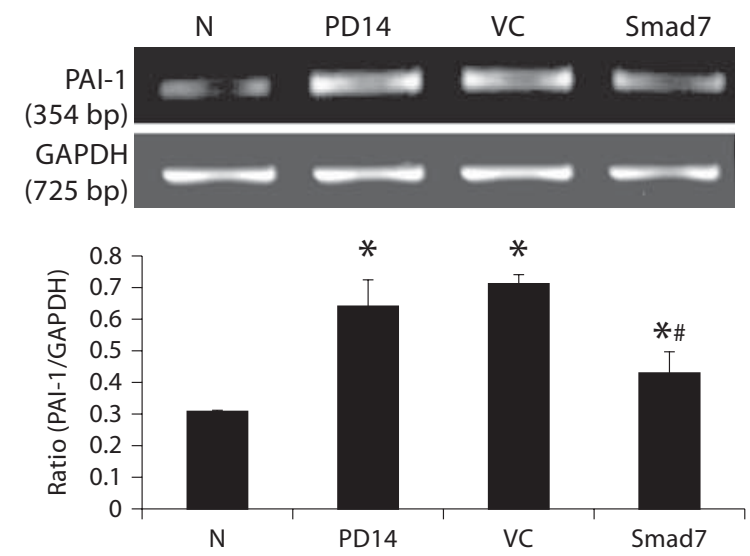

C Confocal microscopy
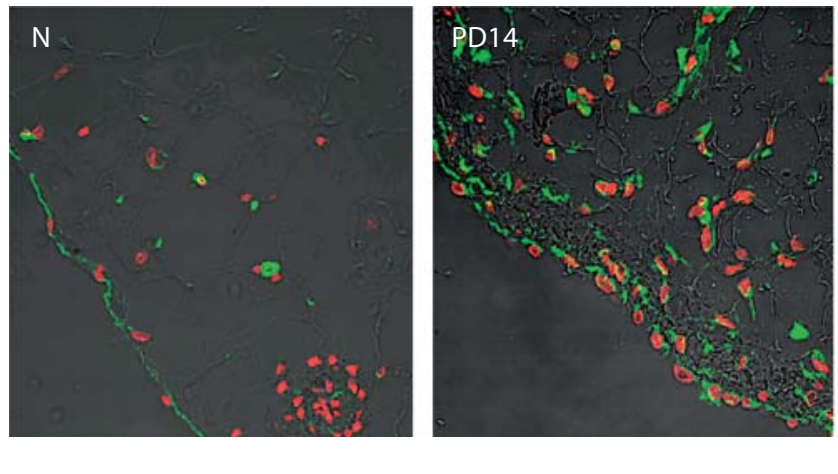

B Western blot

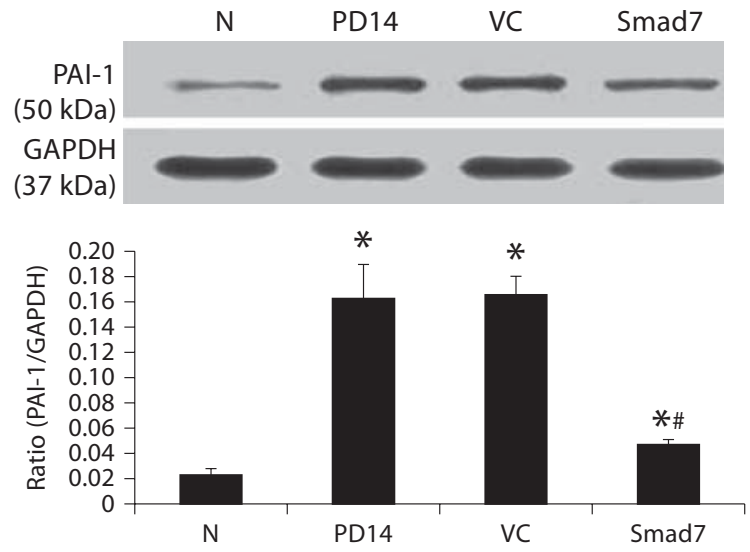

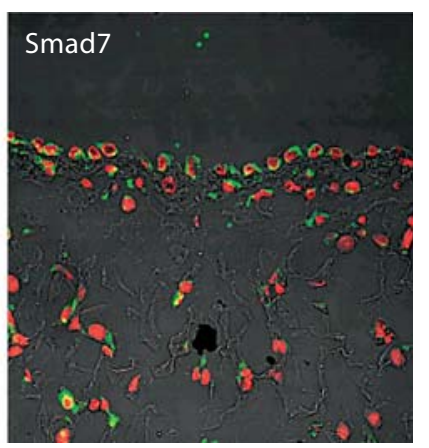

A Western blot
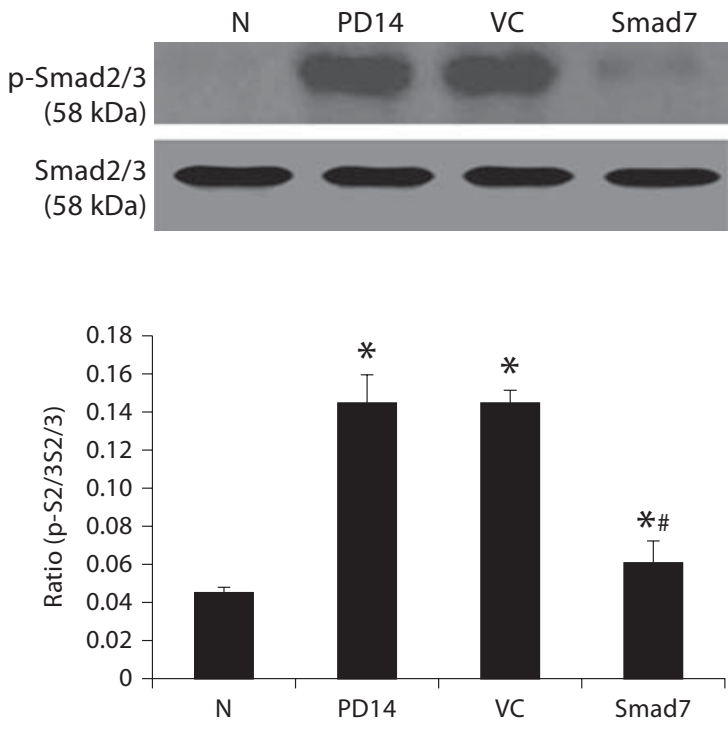

B Confocal microscopy
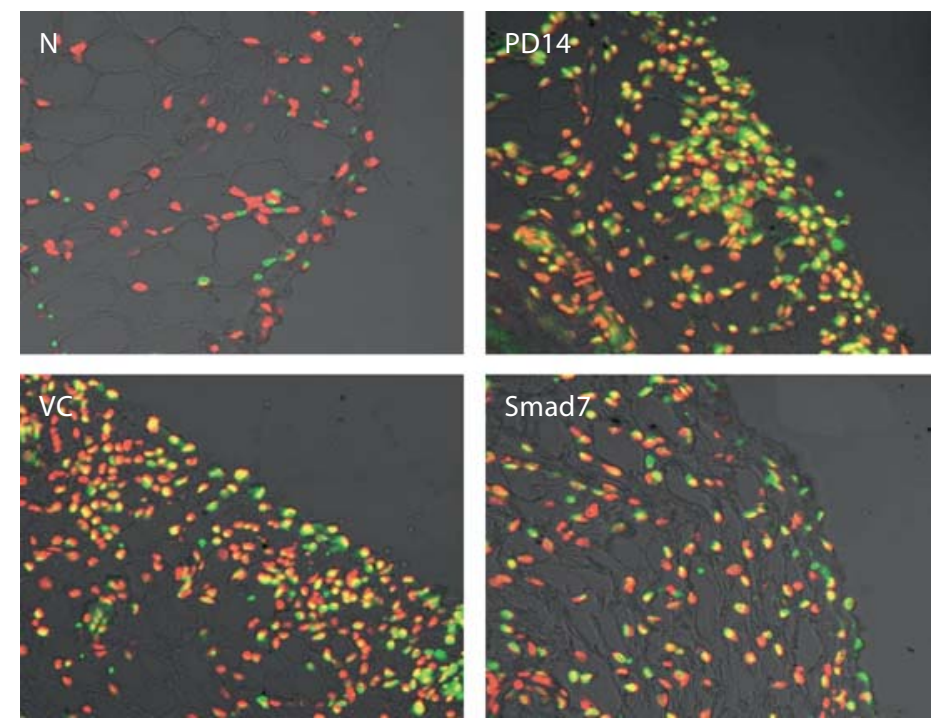

8 
TGF- $\beta$ hyperactivity is recognized as the driving force of the fibrogenic response to injury, and anti-TGF- $\beta$ strategies were successfully used to counteract this process in different issues [23]. It is known that Smad7 functions to negatively regulate TGF- $\beta$ signaling and is thought to be a therapeutic agent for renal fibrosis [24]. The anti-fibrotic effect of Smad7 has been shown by many studies with Smad7 gene expression in vitro and gene transfer in rat models of obstructive kidney disease, remnant kidney disease, and autoimmune nephritis [14-19]. Recently, we and other investigators have also shown that overexpression of peritoneal Smad7 is able to prevent PDinduced peritoneal fibrosis in both uremic or non-uremic rat models [20-22]. However, it remains unclear whether Smad7 has a therapeutic effect on established peritoneal fibrosis, which was addressed in this study in an established model of peritoneal fibrosis induced by a chronic infusion of PD fluids. Inhibition of peritoneal fibrosis on the established model of peritoneal fibrosis demonstrated the therapeutic potential of Smad7 gene therapy for peritoneal fibrosis that mimics clinical conditions.

It has been previously shown that inhibition of TGF$\beta /$ Smad signaling is a key mechanism by which Smad7 gene transfer prevents peritoneal fibrosis in a rat model of PD [20-22]. A similar mechanism was also found in the present study on the established peritoneal fibrosis. This was supported by the findings that overexpression of Smad7 reduced levels of phosphorylated Smad2/3 and expression of TGF- $\beta 1$, thereby improving the severity of peritoneal fibrosis and peritoneal dysfunction.
Consistent with the previous findings [21], blockade of peritoneal PAI-1 expression could be another mechanism of the reversal of peritoneal fibrosis by Smad7. It has been shown that glucose induces peritoneal fibrosis by stimulating PAI-1 expression [25], which can be blocked by anti-TGF- $\beta$ strategies [26]. It is also known that PAI-1 is a component of ECM and a target gene of TGF- $\beta$ because several Smad-binding boxes (CAGA) are in the PAI-1 promoter [27]. Therefore, activation of TGF- $\beta /$ Smad signaling in response to PD fluids may result in upregulation of PAI-1 during peritoneal fibrosis, which can be blocked by overexpression of Smad7 as seen in this and previous studies [21].

In summary, Smad7 gene transfer is able to inhibit the established peritoneal fibrosis induced by a chronic infusion of PD fluids in rats. Blockade of activated TGF- $\beta$ / Smad signaling and its downstream mediators such as PAI-1 may be a key mechanism of inhibition of the established peritoneal fibrosis by Smad7. Results from this study indicate overexpression of Smad7 might merit further clinical investigation for the treatment of peritoneal fibrosis in CAPD patients.

\section{Acknowledgments}

This work was supported by research grants from the Guangdong province Natural Science Foundation of China (2003-36640) and the Research Grants Council of Hong Kong (RGC, GRF767608).
Fig. 7. Smad7 gene transfer blocks PAI-1 mRNA and protein expression in a rat model of peritoneal fibrosis. $\mathrm{N}=$ Normal animals; PD14 $=$ PD rats on day 14 before treatment; $\mathrm{VC}=\mathrm{PD}$ rats treated with empty plasmids on day $28 ; \mathrm{Smad} 7=\mathrm{PD}$ rats treated with Smad7 gene transfer on day 28. All analyses show that Smad7 treatment inhibits PAI-1 mRNA and protein expression in the established model of peritoneal fibrosis. Data represent mean \pm SD for groups of 6 rats. ${ }^{*} \mathrm{p}<0.05$ compared to the age-matched normal control; ${ }^{*} \mathrm{p}<0.05$ compared to both PD14 and VC animals. A RT-PCR. B Western blot analysis. C Confocal microscopy. Nuclei are counterstained with DAPI (red). $\times 400$.
Fig. 8. Smad7 gene transfer blocks $S m a d 2 / 3$ activation in a rat model of established peritoneal fibrosis. A Western blot analysis. $\mathrm{N}=$ Normal animals; PD14 = PD rats on day 14 before treatment; $\mathrm{VC}=\mathrm{PD}$ rats treated with empty plasmids on day $28 ; \mathrm{Smad} 7=\mathrm{PD}$ rats treated with Smad7 gene transfer on day 28. All analyses show that Smad7 gene transfer blocks activation of TGF-/Smad signaling in the established model of peritoneal fibrosis. Data represent mean \pm SD for groups of 6 rats. ${ }^{*} p<0.05$ compared to the agematched normal control; ${ }^{*} \mathrm{p}<0.05$ compared to both PD14 and VC animals. B Confocal microscopy. Nuclei are counterstained with DAPI (red). $\times 400$. 


\section{References}

1 Flessner MF: The effect of fibrosis on peritoneal transport; in Ronco C, Dell'Aquila R, Rodighiero MP (eds): Peritoneal Dialysis: A Clinical Update. Contrib Nephrol. Basel, Karger, 2006, vol 150, pp 174-180.

-2 Plum J, Hermann S, Fussholler A, et al: Peritoneal sclerosis in peritoneal dialysis patients related to dialysis settings and peritoneal transport properties. Kidney Int 2001;(suppl 78):S42-S47.

-3 Musi B, Braide M, Carlsson O, et al: Biocompatibility of peritoneal dialysis fluids: longterm exposure of nonuremic rats. Perit Dial Int 2004;24:37-47.

4 Faull RD: Bad and good growth factors in the peritoneal cavity. Nephrology (Carlton) 2005; 10:234-239.

5 Margetts PJ, Oh KH, Kolb M: Transforming growth factor-beta: importance in long-term peritoneal membrane changes. Perit Dial Int 2005;25(suppl 3):S15-S17.

-6 Medcalf JF, Walls J, Pawluczyk IZ, et al: Effects of glucose dialysate on extracellular matrix production by human peritoneal mesothelial cells (HPMC): the role of TGF- $\beta$. Nephrol Dial Transplant 2001;16:18851892.

7 Yung S, Chen XR, Tsang RC, et al: Reduction of perlecan synthesis and induction of TGF$\beta 1$ in human peritoneal mesothelial cells due to high dialysate glucose concentration: implication in peritoneal dialysis. J Am Soc Nephrol 2004; 15:1178-1788.

-8 Lai KN, Lai KB, Lam CW, et al: Changes of cytokine profiles during peritonitis in patients on continuous ambulatory peritoneal dialysis. Am J Kidney Dis 2000;35:644-652.

$\checkmark 9$ Lin CY, Chen WP, Yang LY, Chen A, Huang $\mathrm{TP}$, et al: Persistent transforming growth factor-beta 1 expression may predict peritoneal fibrosis in CAPD patients with frequent peritonitis occurrence. Am J Nephrol 1998; 18:513-519.
10 Margetts PJ, Kolb M, Galt T, Hoff CM, Shockley TR, Gauldie J: Gene transfer of transforming growth factor-betal to the rat peritoneum: effects on membrane function. J Am Soc Nephrol 2001;12:2029-2039.

11 Margetts PJ, Bonniaud P, Liu L, et al: Transient overexpression of TGF-betal induces epithelial mesenchymal transition in the rodent peritoneum. J Am Soc Nephrol 2005; 16 : 425-436.

12 Kretzschmar M, Massagué J: SMADs: mediators and regulators of TGF-beta signaling. Curr Opin Genet Dev 1998;8:103-111.

13 Kavsak P, Rasmussen RK, Causing CG, et al: Smad7 binds to Smurf2 to form an E3 ubiquitin ligase that targets the TGF beta receptor for degradation. Mol Cell 2000;6:13651375.

14 LiJH, Zhu HJ, Huang XR, et al: Smad7 inhibits fibrotic effect of TGF-beta on renal tubular epithelial cells by blocking Smad2 activation. J Am Soc Nephrol 2002;13:1464-1472.

$\checkmark 15$ Chen R, Huang C, Morinelli TA, et al: Blockade of the effects of TGF- $\beta 1$ on mesangial cells by overexpression of Smad7. J Am Soc Nephrol 2002;13:887-893.

16 Terada Y, Hanada S, Nakao A, et al: Gene transfer of Smad7 using electroporation of adenovirus prevents renal fibrosis in postobstructed kidney. Kidney Int 2002;61 (suppl):S94-S98.

17 Lan HY, Mu W, Tomita N, et al: Inhibition of renal fibrosis by gene transfer of inducible Smad7 using ultrasound-microbubble system in rat UUO model. J Am Soc Nephrol 2003; 14:1535-1548.

18 Hou CC, Wang W, Huang XR, et al: Ultrasound-microbubble-mediated gene transfer of inducible Smad7 blocks transforming growth factor-beta signaling and fibrosis in rat remnant kidney. Am J Pathol 2005;166: 761-771.
19 Ka SM, Huang XR, Lan HY, et al: Smad7 gene therapy ameliorates an autoimmune crescentic glomerulonephritis in mice. J Am Soc Nephrol 2007;18:1777-1788.

20 Guo H, Leung JC, Lam MF, et al: Smad7 transgene attenuates peritoneal fibrosis in uremic rats treated with peritoneal dialysis. J Am Soc Nephrol 2007;18:2689-2703.

21 Nie J, Dou X, Hao W, et al: Smad7 gene transfer inhibits peritoneal fibrosis. Kidney Int 2007;72:1336-1344.

22 Nie J, Hao W, Dou X, et al: Effects of Smad7 overexpression on peritoneal inflammation in a rat peritoneal dialysis model. Perit Dial Int 2007;27:580-588.

23 Wang W, Koka V, Lan HY: Transforming growth factor-beta and Smad signalling in kidney diseases. Nephrology (Carlton) 2005; 10:48-56.

24 Lan HY: Smad7 as a therapeutic agent for chronic kidney diseases. Front Biosci 2008; 13:4984-4992.

25 Higuchi C, Tanihata Y, Nishimura H, et al: Effects of glucose and plasminogen activator inhibitor-1 on collagen metabolism in the peritoneum. Ther Apher Dial 2005;9:173181.

26 Dennler S, Itoh S, Vivien D, et al: Direct binding of Smad 3 and Smad4 to critical TGF beta-inducible elements in the promoter of human plasminogen activator inhibitortype 1 gene. EMBO J 1998;17:3091-3100.

27 Liu F, Liu H, Peng Y, et al: Inhibition of transforming growth factor beta (TGFbeta1) expression and extracellular matrix secretion in human peritoneal mesothelial cells by pcDU6 vector-mediated TGFbetal shRNA and by pcDNA3.1(-)-mediated antisense TGFbeta1 RNA. Adv Perit Dial 2005;21:4152. 